\title{
Detection of T. vaginalis, M. hominis, M. genitalium, C. trachomatis, N. gonorrhoeae and U. urealyticum using Multiplex PCR
}

\author{
Tamara Brunelli, Roberto Degl'Innocenti, Patrizia Miglietta, Loredana Ortega De Luna, \\ Elisa Rovinati, Stefania Apolito, Antonella Conti, Patrizia Casprini \\ U.O. Analisi Chimico Cliniche, Ospedale Misericordia e Dolce, USL4, Prato
}

Key words: multiplex PCR, pathogens, sexually transmitted diseases

Ricerca di T. vaginalis, M. hominis, M. genitalium, C. trachomatis, N. gonorrhoeae e U. urealyticum mediante l'uso di PCR Multiplex

\section{SUMMARY}

Intoduction. The sexually transmitted diseases include a large group of infections affecting both the sexes. In this study we evaluated the prevalence of Trichomonas vaginalis, Mycoplasma hominis, Mycoplasma genitalium, Chlamydia trachomatis, Neisseria gonorrhoeae and Ureaplasma urealyticum in the Prato area during the period September 2010 - July $201 \mathrm{I}$.

Methods. We analysed different kind of samples (urine, endocervical swabs, urethral swabs, seminal fluids) from hospitalized patients or referred to the Prato clinic subjects. The DNA was obtained using EZI-DNA extraction kit and EZI instrument. The DNA was then amplified using the Seeplex STD6 kit (Seegene, Korea), identifying multiple pathogens simultaneously (T. vaginalis, M. hominis, M. genitalium, C. trachomatis, N. gonorrhoeae e $U$. urealyticum). The revelation was performed by electrophoresis on microchip (instrument Multina, Shimadzu, Japan).

Results. II 36 samples from Italian and foreign patients were examined: 876 were endocervical swabs (77\%), 103 urethral swabs (9\%), 103 seminal fluids (9\%), and 54 urines (5\%). The number of females was higher than males [894 (78.7\%) vs $242(21.3 \%)$; the mean age of females was $37.0 \pm 11.6$ years, whereas that of males was $4 I .5 \pm 12.63$ years. The prevalence of urogenital pathogens was: I 5 positive samples for T. vaginalis (I.3\%), 56 for M. hominis (4.9\%), I 3 for M. genitalium (I.I\%), 28 for C. trachomatis (2.5\%), 8 for N. gonorrhoeae $(0.7 \%)$ and 87 for U. urealyticum (7.7\%). Among all positive, 25 subjects were positive for more than one pathogen and in particular: one was positive for the presence of 4 pathogens, five presented 3 pathogens simultaneously and the remaining nineteen for 2 pathogens.

Conclusions. This study provides data on the prevalence of sexually transmitted diseases in the hospital of Prato.

\section{INTRODUZIONE}

Le malattie a trasmissione sessuale comprendono un ampio gruppo di infezioni che interessano entrambi i sessi. L'esordio, di solito acuto, può sfociare successivamente in una situazione cronica. Inoltre, può anche accadere che il singolo paziente sia affetto da una sindrome ad eziologia multipla (2).

La ricerca simultanea ed in tempi brevi di più patogeni trasmissibili per via sessuale è di notevole importanza sia per la prevenzione della diffusione di tali malattie, sia per la riduzione delle complicanze ad esse associate.

In questo studio, mediante 1'uso di PCR multiplex, è stata valutata la prevalenza di Trichomonas vaginalis, Mycolplasma hominis, Mycoplasma genitalium, Chlamydia trachomatis, Neisseria gonorrhoeae e Ureaplasma urealyticum in pazienti afferenti nel periodo settembre 2010luglio 2011 all'ospedale di Prato.

\section{MATERIALI E METODI}

Sono stati analizzati un totale di 1136 soggetti afferenti all'ospedale di Prato. I 1136 campioni raccolti erano così suddivisi: 54 urine $(5 \%), 876$ tamponi endocervicali (76\%), 103 tamponi uretrali (9\%) e 103 liquidi seminali (9\%). Tali campioni provenivano da pazienti italiani e/o stranieri afferenti ad ambulatori e reparti dell' ospedale di Prato (USL4, Toscana).

I tamponi endocervicali ed i tamponi uretrali sono stati raccolti in contenitori Copan (a.d.a s.r.l., Milano) contenenti liquido di trasporto.

Urine e liquido seminale sono stati raccolti in pro-

\section{Corresponding author: Tamara Brunelli}

U.O. Analisi Chimico Cliniche, Ospedale Misericordia e Dolce, USL4

Piazza dell'Ospedale, 5 - 59100 Prato - Tel.: 0574 4347। 9

E-mail: tbrunelli@usl4.toscana.it 
vette sterili (Sputocol, Evergreen Sc., USA) prive di additivi.

Il DNA genomico è stato ottenuto, a partire dai campioni, utilizzando il kit di estrazione EZ1DNA e l'apparecchio EZ1 (Qiagen, Germania). L'amplificazione del DNA è stata eseguita con il kit Seeplex STD6 (Seegene, Korea) seguendo le indicazioni del produttore. Tale kit permette l'amplificazione contemporanea dei patogeni $T$. vaginalis, M. hominis, M. genitalium, C. trachomatis, $N$. gonorrhoeae ed $U$. urealyticum.

La rivelazione è stata eseguita mediante elettroforesi su microchip (apparecchio Multina, Shimadzu, Giappone).

Il kit prevede l'amplificazione della $\beta$-globina (controllo interno) per la rilevazione, nei campioni, della presenza di DNA umano (Samra 2011).

\section{RISULTATI}

Sono stati esaminati un totale di 1136 soggetti afferenti all'ospedale di Prato (interni ed esterni), in maggioranza donne (il $78.7 \%$ dei soggetti).

Il numero complessivo di femmine e maschi, nonché le loro caratteristiche di età media e range di età sono riportati in Tabella 1. L'età media delle femmine è risultata significativamente inferiore rispetto a quella dei maschi $(p=0.000)$ (Tabella 1).

In Tabella 2 sono riportati i valori percentuali di prevalenza, in base al sesso, dei differenti patogeni identificati. Nei soggetti in studio la prevalenza delle specie $M$. hominis ed $U$. urealyticum è risultata significativamente più elevata nelle femmine che nei maschi. Il patogeno $N$. gonorrhoeae ha mostrato, al contrario, una prevalenza significativamente più elevata nei
Tabella I. Soggetti esaminati.

\begin{tabular}{|c|c|c|c|}
\hline & Maschi & Femmine & Totale \\
\hline $\mathbf{N}^{\circ}$ & $242(21,3 \%)$ & 894 (78.7\%) & 1136 \\
\hline \multirow{2}{*}{ Età (anni) } & Media 4I.5 \pm 12.63 & Media $37.0 \pm I$ I.6 & Media $37.9 \pm \mid 2.0$ \\
\hline & Range I 8-80 & Range 16-86 & Range 16-86 \\
\hline
\end{tabular}

Tabella 2. Prevalenza dei patogeni analizzati nella popolazione studiata.

\begin{tabular}{lllll}
\hline T. vaginalis & Maschi & Femmine & Totali & Significatività (S) \\
\hline M. hominis & $2(0.8 \%)$ & $13(1.4 \%)$ & $15(1.3 \%)$ & Non S \\
\hline M. genitalium & $4(1.7 \%)$ & $\mathbf{5 4}(1.0 \%)$ & $56(4.9 \%)$ & $p=0.000$ \\
\hline C. trachomatis & $10(0.4 \%)$ & $18(2.0 \%)$ & $28(2.5 \%)$ & Non S \\
\hline N. gonorrhoeae & $5(2.1 \%)$ & $3(0.33 \%)$ & $8(0.7 \%)$ & $p=0.013$ \\
\hline U. urealyticum & $6(2.5 \%)$ & $81(9.1 \%)$ & $87(7.7 \%)$ & $P=0.000$ \\
\hline
\end{tabular}

Tabella 3. Soggetti positivi per più di un microrganismo.

\begin{tabular}{ll}
\hline Organismi & $\mathbf{N}^{\circ}$ Pazienti \\
\hline T. vaginalis + M. hominis & 3 \\
\hline T. vaginalis + U. urealyticum & $\mathrm{I}$ \\
\hline T. vaginalis + U. urealyticum + M. hominis & $\mathrm{I}$ \\
\hline T. vaginalis + M. hominis + M. genitalium & $\mathrm{I}$ \\
\hline T. vaginalis + M. hominis + M. genitalium + C. trachomatis & $\mathrm{I}$ \\
\hline
\end{tabular}

\begin{tabular}{ll}
\hline C. trachomatis + M. genitalium & 2 \\
\hline C. trachomatis + M. hominis + U. urealyticum & 2 \\
\hline M. hominis + U. urealyticum & 12 \\
\hline M. hominis + N. gonorrheae & I \\
\hline M. hominis + M. genitalium + U. urealyticum & $\mathrm{I}$ \\
\hline
\end{tabular}

Tabella 4. Prevalenza di positivi suddivisi per fasce di età e sesso.

\begin{tabular}{|c|c|c|c|c|c|}
\hline \multirow[t]{2}{*}{ GERME } & \multirow[t]{2}{*}{ sesso } & $\leq 25$ anni & 26-50 anni & \multicolumn{2}{|l|}{$>50$ anni } \\
\hline & & I7M I29F & $169 \mathrm{M} 659 \mathrm{~F}$ & $56 \mathrm{M} \quad \mathrm{l} 06 \mathrm{~F}$ & \\
\hline \multirow[t]{2}{*}{ T.vaginalis } & M & 0 & $I(0.6 \%)$ & $I(I .8 \%)$ & \\
\hline & $\mathbf{F}$ & 0 & I I(I.7\%) & $2(1.9 \%)$ & \\
\hline \multirow[t]{2}{*}{ M. hominis } & $M$ & 0 & $2(2.4 \%)$ & 0 & \\
\hline & $\mathbf{F}$ & $13(10 \%)$ & $33(5 \%)$ & $8(7.5 \%)$ & \\
\hline \multirow[t]{2}{*}{ M. genitalium } & M & 0 & $4(2.4 \%)$ & 0 & \\
\hline & $\mathbf{F}$ & $2(1.6 \%)$ & 7 (1.1\%) & 0 & \\
\hline \multirow[t]{2}{*}{ C. trachomatis } & $M$ & $I(5.8 \%)$ & $7(4 \%)$ & $2(3.6 \%)$ & \\
\hline & $\mathbf{F}$ & $9(6.5 \%)$ & $8(1.2 \%)$ & $I(0.9 \%)$ & $p=0.000$ \\
\hline \multirow[t]{2}{*}{ N. gonorrheae } & $M$ & $I(5.8 \%)$ & $4(2.4 \%)$ & 0 & \\
\hline & $\mathbf{F}$ & 0 & $2(0.3 \%)$ & I (0.9\%) & \\
\hline \multirow[t]{2}{*}{ U. urealyticum } & $M$ & $I(5.8 \%)$ & $5(3.0 \%)$ & 0 & \\
\hline & $\mathbf{F}$ & $24(19.0 \%)$ & $5 \mathrm{I}(7.7 \%)$ & $6(5.7 \%)$ & $p=0.000$ \\
\hline
\end{tabular}

Legenda: $M=$ maschi; $F=$ femmine maschi $(p=0.013)$. distribuzione nei due sessi.

distinguere un totale di $182(88 \%)$ soggetti con infezioni provocate da un singolo patogeno; 25 soggetti erano invece positivi per la presenza di due o più patogeni (Tabella 3 ). 
È stato inoltre possibile osservare un'associazione tra la presenza della specie $T$. vaginalis e quella di $M$. hominis; la specie $M$. hominis è stata infatti identificata nel $33 \%$ dei soggetti positivi per $T$. vaginalis mentre era presente solo nel $4.5 \%$ dei pazienti non colonizzati da T. vaginalis (Figura I).

Raggruppando i pazienti in base all'età, si può osservare come il gruppo più numeroso sia quello compreso fra i 26 ed i 50 anni, comprendente 828 soggetti.

Nelle donne è stata osservata una distribuzione significativamente diversa, per fascia di età, di C. trachomatis ed U. urealyticum (Tabella 4).

Una più elevata prevalenza per entrambi i microrganismi è stata osservata all'interno del gruppo delle donne giovani $(<25$ anni); la prevalenza diminuiva poi in base all'aumentare dell'età per entrambi i sessi. Per quanto riguarda il T. vaginalis la frequenza di isolamento aumentava parallelamente all'età in entrambi i sessi.

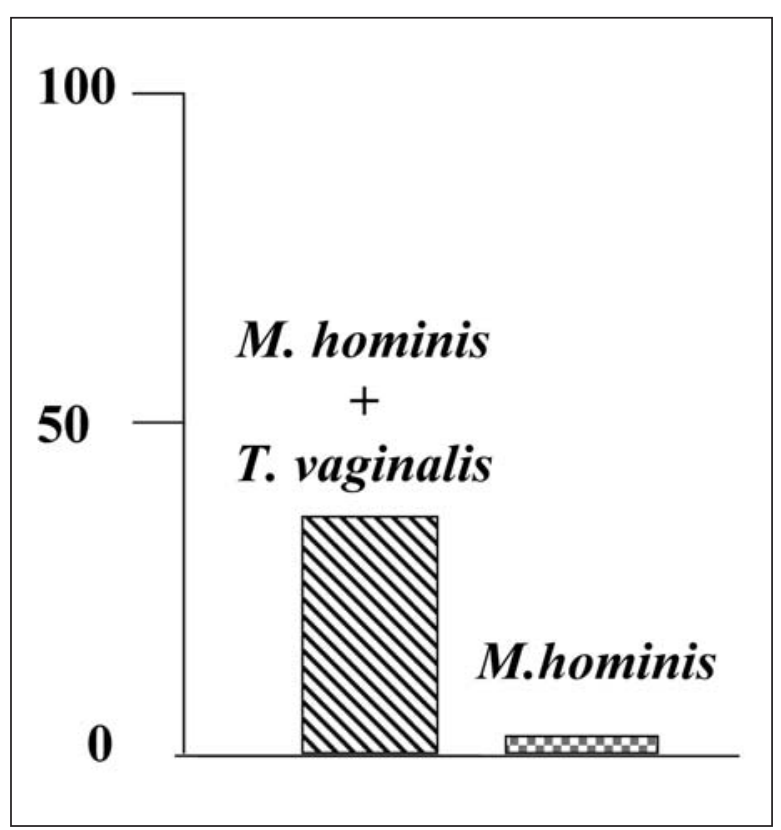

Figura I. Associazione tra T. vaginalis e M. hominis.

\section{DISCUSSIONE}

In questo studio è stata valutata, con un metodo molecolare standardizzato introdotto nel nostro laboratorio nel settembre 2010 (4), la contemporanea presenza di sei patogeni urogenitali, nello stesso campione, in 1136 pazienti.

La presenza degli stessi microrganismi era stata valutata in precedenza con metodi diversi (coltura, PCR, immunocromatografia). L'impiego di un approccio molecolare consente tuttavia di risparmiare tempo e risorse economiche.

Dati di letteratura indicano come patogeni diversi, quali ad esempio $T$. vaginalis e $M$. hominis, sebbene causino sintomi simili, rispondono a terapie differenti; è dunque importante poterne rilevare anche la contemporanea presenza per risolvere definitivamente l'infezione.

Nei soggetti analizzati in questo studio è stata riscontrata un'associazione tra la presenza di $T$. vaginalis e quella di $M$. hominis; questo dato è in accordo con quanto pubblicato recentemente in pazienti italiani (3).

Tra le infezioni batteriche, C. trachomatis, U. urealyticum e M. genitalium sono stati associati al verificarsi di aborti spontanei ricorrenti; in questi casi una diagnosi in tempi brevi può portare ad un intervento terapeutico più efficace.

Raggruppando i pazienti in base all'età, abbiamo evidenziato una maggiore prevalenza di $C$. trachomatis nelle donne giovani ( $<25$ anni).

Questo è in accordo con uno studio che riferiva una più elevata prevalenza $(7.8 \%)$ di questo patogeno in donne giovani, sintomatiche e non, nel Nord Italia (1).

Uno studio condotto nella zona di Torino su donne sintomatiche, ha evidenziato che la prevalenza di C. trachomatis mostrava un picco nei pazienti con meno di 25 anni e che la frequenza di $T$. vaginalis aumentava con l'età (3.3\% tra i 55-65 anni); la più elevata prevalenza di $C$. trachomatis riscontrata in confronto ai dati ottenuti nel presente studio è probabilmente dovuta alla selezione, nello studio torinese, di pazienti con sintomi (5).

In conclusione, l'uso di PCR multiplex nel nostro laboratorio per la ricerca di patogeni urogenitali si è rivelato di particolare utilità:

- sia in termini di risparmio di risorse economiche, grazie alla riduzione del numero dei test da eseguire; la ricerca di C. trachomatis veniva eseguita infatti in precedenza con PCR, quella di T. vaginalis con metodo immunocromatografico, e quelle di M. hominis, U. urealyticum e $N$. gonorrhoeae con metodo colturale,

- sia perché ha permesso di ampliare il pannello dei patogeni analizzati con l'introduzione della ricerca di $M$. genitalium senza incrementare il carico di lavoro,

- sia perché nella nostra tipologia di popolazione alcune specie patogene possono essere presenti contemporaneamente nello stesso paziente e risulta quindi utile l'adozione di un unico test che permetta una loro simultanea individuazione.

\section{BIBLIOGRAFIA}

1. Andreoni S, Brunelli MG, Camaggi A, et al. Incidence of Chlamydia trachomatis infections in the provinces of Novara and VCO: one year of observation. Micr Med. 2009; 24: 239

2. Casari E, Ferrario A, Marenghi E, et al. Gardnerella, Trichomonas vaginalis, Candida, Chlamydia trachomatis, Mycoplasma hominis and Ureaplasma urealyticum in the genital discharge of symptmatic fertile 
and asymptomatic infertile women. New Microbiologica. 2010; 33: 69-76.

3. Diaz N, Dessì D, Dessole S, et al. Rapid detection of coinfection by Trichomonas vaginalis, Mycoplasma hominis, and Ureaplasma urealyticum by a new multiplex polymerase chain reaction. Diagn Micr Inf Dis. 2010; 67: 30-6.

4. Samra Z, Rosenberg S, Madar-Shapiro L. Direct simultaneous detection of 6 sexually transmitted pathogens from clinical specimens by multiplex polymerase chain reaction and auto-capillary electrophoresis. Diagn Micr Inf Dis. 2011; 70 (1): 17-21.

5. Tibaldi C, Cappello N, Latino MA, et al. Vaginal and endocervical microorganisms in symptomatic and asymptomatic non-pregnant females risk factors and rate of occurrence. Clin Microbiol Infect. 2009; 15 (7): 670-9. 